\title{
Equilíbrio estático de indivíduos com neuropatia periférica diabética
}

\author{
Static balance in individuals with diabetic peripheral neuropathy \\ Kelson Luiz da Silva Sales', Leonardo Alves de Souza', Vinicius Saura Cardoso²
}

RESUMO I O diabetes mellitus (DM) é uma das enfermidades crônicas mais diagnosticadas nos últimos anos. A neuropatia diabética periférica (NP) é a complicação mais prevalente dessa doença, atingindo até $80 \%$ dos diabéticos, podendo modificar o equilíbrio. Este estudo teve por objetivo comparar o equilíbrio estático de indivíduos diabéticos neuropatas, diabéticos não neuropatas e indivíduos sem DM e averiguar a influência da visão nessa situação. Foram avaliados 30 indivíduos, divididos em três grupos diferentes: GC, 10 não diabéticos (média de idade 55,5 \$9,72 anos); GD: 10 diabéticos sem NP (54,4 7,76 anos); e GNP, 10 diabéticos com NP (60,4ะ5,35 anos). Cada indivíduo foi filmado nos planos frontal e sagital, com e sem visão, avaliado quadro a quadro no software Free Video to JPG Converter ${ }^{\circledR}$, para a seleção dos momentos de maiores oscilações. As imagens foram quantificadas através da Biofotogrametria Computadorizada, utilizando-se o software SAPO. Os dados foram tratados estatisticamente no software Graph Pad Prism (versão 5). Os resultados evidenciam que o grupo GNP apresentou diferenças estatisticamente significantes nas amplitudes de oscilações no plano frontal e sagital, nas condições com e sem visão, quando comparado com os outros grupos ( $p=0,0001)$. Mediante os resultados, concluiu-se que a NP influencia negativamente na manutenção do equilíbrio estático, principalmente sem visão.

Descritores I diabetes mellitus; neuropatias diabéticas; equilíbrio postural.
ABSTRACT I Diabetes mellitus (DM) is one of most chronic diseases diagnosed in recent years. Peripheral diabetic neuropathy (PN) is the most prevalent complication of the disease, reaching $80 \%$ of diabetics and it may modify the balance. This study intended to compare the static equilibrium of neuropathic diabetics, diabetic nonneuropathic and subjects without DM and investigate the influence of vision in this situation. Thirty subjects were evaluated, divided into three different groups: GC, 10 non-diabetic (average age 55.5 \pm 9.72 years); GD, 10 diabetic without NP (54.4 \pm 7.76 years); and GNP, 10 diabetic with NP $(60.4 \pm 5.35$ years). All subjects were filmed in the frontal and sagittal planes, with and without vision, and their movements were evaluated frame by frame in the software Free Video to JPG Converter ${ }^{\circledR}$ to select the moments of greatest oscillation. The images were quantified through computerized biophotogrammetry using the software SAPO. Data were statistically analyzed in Graph Pad Prism Software (version 5). The results pointed that the GNP group showed statistically significant differences in the amplitudes of oscillations in the frontal and sagittal, both with and without vision, compared with other groups ( $p=0.0001$ ). With the results it can be concluded that NP has a negative influence on the maintenance of static balance, particularly without vision.

Keywords I diabetes mellitus; diabetic neuropathies; postural balance.

Estudo desenvolvido no Laboratório de Biofotogrametria do Curso de Fisioterapia da Universidade Federal do Piauí, Campus Ministro Reis Velloso - Parnaíba (PI), Brasil.

${ }^{1}$ Acadêmico do Curso de Fisioterapia da Universidade Federal do Piauí (UFPI) - Parnaíba (PI), Brasil.

${ }^{2}$ Professor do Curso de Fisioterapia da UFPI - Parnaíba (PI), Brasil. 


\section{INTRODUÇÃO}

O diabetes mellitus (DM) é uma das enfermidades crônicas mais diagnosticadas nos últimos anos. Há cerca de 170 milhões de indivíduos diabéticos no mundo e há uma previsão de aumento de $114 \%$ até $2030^{1}$.

A neuropatia diabética periférica (NP) é a complicação mais prevalente dessa doença ${ }^{2}$, é progressiva e irreversível $^{3,4}$, atingindo até $80 \%$ dos diabéticos ${ }^{5,6}$. Estudos anteriores mostram que a NP leva a perda das informações sensoriais cutâneas nos pés, podendo ocasionar transtornos da estrutura osteoarticular dessa região, além de contribuir para modificar a marcha, o equilíbrio estático e dinâmico ${ }^{7}$. Kandel et al. definiram o equilíbrio estático como sendo a manutenção do corpo em uma posição em que seu centro de massa permaneça dentro de uma base de suporte (pés), sem que haja oscilações significantes, ou seja, nos limites da estabilidade ${ }^{8}$.

A manutenção do equilíbrio do corpo humano depende da coordenação intrínseca do sistema vestibular, da visão e das informações táteis e proprioceptivas. Esses componentes trabalham de forma integrada e complementar e qualquer alteração em um, ou mais, desses sistemas resultam em instabilidade postural ${ }^{9,10}$ e, consequentemente, aumentam o risco de quedas, lesões cutâneas, fraturas e imobilizações prolongadas, levando assim a redução da qualidade de vida dessa população $0^{11-13}$.

Estudos anteriores ${ }^{14-18}$ encontraram que indivíduos diabéticos neuropatas demonstram déficts de equilíbrio. Embora a NP seja comumente pensada como a causa dessa instabilidade postural, há alguma evidência de que o diabetes, por si só, pode ter também um impacto negativo no controle postural em condições estressantes $^{17,19-21}$. Devido ao crescimento dessa evidência, mais pesquisas explorando o impacto do diabetes e da NP no controle postural são necessárias. Assim, o objetivo deste estudo foi comparar o equilíbrio estático de indivíduos diabéticos neuropatas, diabéticos não neuropatas e indivíduos sem DM e averiguar a influência da visão nessa situação.

\section{METODOLOGIA}

Este é um estudo analítico comparativo sobre o efeito da NP no equilíbrio estático de indivíduos diabéticos. A amostra foi do tipo de conveniência, composta por 32 indivíduos (22 mulheres e 10 homens), recrutados de uma Unidade Básica de Saúde da cidade de Parnaíba (PI). Destes, 22 eram diabéticos e 10 não diabéticos, divididos em 3 grupos: GC (controle), formado por 10 indivíduos não diabéticos; GD, formado por 10 indivíduos diabéticos sem NP; e GNP, formado por 10 diabéticos com NP. A presença de DM foi verificada segundo critérios da American Diabetes Association ${ }^{22}$. Foram excluídos dois indivíduos que se enquadraram pelo menos em um dos seguintes critérios de não seleção: diagnóstico clínico de doenças neurológicas que afetam a postura e que não sejam causadas pelo DM, vestibulopatias, úlceras plantares, alterações ortopédicas ou posturais.

Após aprovação deste estudo pelo Comitê de Ética e Pesquisa da Universidade Federal do Piauí (UFPI), sob no 0403.0.045.000-10, todos os participantes assinaram um Termo de Consentimento Livre e Esclarecido. A coleta ocorreu no Laboratório de Biofotogrametria da UFPI, campus Ministro Reis Velloso.

\section{Procedimentos}

Os participantes foram submetidos a uma avaliação inicial, contendo dados pessoais e antropométricos (massa corpórea, estatura e índice de massa corpórea-IMC) e aspectos relacionados ao diabetes (presença e tipo, tempo de diagnóstico médico). Todos foram pesados em uma balança digital (Welmy ${ }^{\circledR}$ W110h) certificada pelo Instituto Nacional de Metrologia, Normalização e Qualidade Industrial (INMETRO).

Todos os participantes submeteram-se à avaliação da sensibilidade protetora dos pés, realizada por meio do monofilamento Semmes-Weinstein de $10 \mathrm{~g}$ $\left(\right.$ SorriBauru $\left.{ }^{\circledR}\right)$. Inicialmente, foi feita uma aplicação no antebraço para verificar a correta compreensão do participante quanto ao teste a ser realizado. Em segui$\mathrm{da}$, com o paciente com os olhos fechados, pediu-se que relatasse quando sentisse a pressão do monofilamento e o local dessa aplicação. O teste foi padronizado com duas aplicações em cada local e a ordem das aplicações foi randomizada pelo examinador ${ }^{23,24}$. O monofilamento foi aplicado de forma perpendicular à planta dos pés durante $2 \mathrm{~s}$, com força suficiente para curvá-lo, na região do hálux, do segundo dedo, do quinto dedo, na projeção da cabeça do primeiro e quinto metatarso, no médio pé e calcâneo. A perda da sensibilidade protetora foi detectada nos casos de ausência dessa percepção em pelo menos dois dos sete pontos testados ${ }^{23}$. Em seguida, foi realizado o teste de sensibilidade vibratória com um diapasão metálico 
(frequência $128 \mathrm{~Hz}$ ), aplicado perpendicularmente sobre a articulação interfalangeana do hálux e do maléolo medial, com o paciente também de olhos fechados. Nos casos em que o indivíduo não sentiu a vibração, foi verificado o comprometimento da sensibilidade profunda.

Os indivíduos com insensibilidade ao monofilamento e ao diapasão preencheram o questionário Michigan Neuropathy Screening Instrument (MNSI) ${ }^{25}$ a fim de confirmar a NP. Aqueles com pontuação igual ou superior a oito, no MNSI, e déficits de sensibilidade foram classificados como grupo $\mathrm{GNP}^{26}$. Os demais diabéticos, sem NP, formaram o grupo GD.

Após a avaliação sensitiva, foram realizados os testes de equilíbrio. Para verificar as oscilações do corpo em equilíbrio estático, foi utilizado um protocolo de estudo que empregou a Biofotogrametria Computadorizada como instrumento quantificador angular do desvio da linha de equilíbrio, sendo aplicado o teste de Romberg adaptado ${ }^{27}$. Para essa avaliação, foram fixados, nos participantes, marcadores adesivos de $5 \mathrm{~mm}$ na glabela (para avaliação no plano frontal) e no trágus da orelha esquerda (para avaliação no plano sagital). Com o marcador alinhado a um fio de prumo, o qual possuía 2 marcadores, separados um do outro à distância de $20 \mathrm{~cm}$, os indivíduos adotavam posição ortostática por $30 \mathrm{~s}$.

A câmera filmadora (Sony ${ }^{\circledR}$ DCR SX63) foi posicionada em nível e em prumo à distância fixa de 3 $\mathrm{m}$ do voluntário e a $1 \mathrm{~m}$ do solo, para a realização de vídeos de 30 s nos dois planos e em 2 condições: com e sem visão, totalizando 4 filmagens. A ordem das filmagens foi randomizada pelo examinador. $\mathrm{Na}$ condição com visão, foi solicitado ao participante que olhasse para dois alvos circulares amarelos, um na parede frontal (para avaliação no plano frontal) e o outro na parece lateral (para avaliação no plano sagital), ambos na altura dos olhos, para minimizar movimentos indesejados da cabeça. Nas condições sem visão, foram utilizados óculos de natação totalmente vedados, a fim de que o participante não tivesse nenhuma informação visual ${ }^{28}$.

As filmagens foram convertidas em 30 imagens digitais pelo software Free Video to JPG Converter ${ }^{\circledR}$ e, em seguida, avaliadas quadro a quadro para a seleção dos momentos de maiores oscilações. As imagens foram quantificadas em graus, utilizando-se o software $S A P O^{29}$, que mensurou a amplitude da oscilação, tendo como referência angular uma reta traçada do marcador ao vértice formado entre o fio de prumo e uma reta perpendicular ao mesmo, traçada $20 \mathrm{~cm}$ acima do marcador.

\section{Análise dos dados}

Para quantificar os graus da amplitude de oscilação no plano sagital, foi realizada a soma da maior amplitude de oscilação, tanto anterior, quanto posterior e, do plano frontal, a soma das maiores oscilações para direita e para esquerda.

A remoção da informação visual foi proposta para evidenciar a alteração do sistema somatossensorial e a necessidade da visão nos pacientes neuropatas para a manutenção do equilíbrio estático, adotando assim, o cálculo da diferença das oscilações de cada participante com e sem visão ${ }^{28}$, de acordo com a equação abaixo:

$$
\text { Diferença }=\text { com visão }- \text { sem visão }
$$

\section{Análise estatística}

A análise estatística foi realizada através do software Graph Pad Prism (versão 5). Neste estudo o intervalo de confiança foi de $95 \%$, adotando o valor $\mathrm{p}<0,05$ como nível de significância. Foi aplicado o teste $t$ de Student pareado, para a avaliação intragrupos, e análise de variância (One way-ANOVA) seguido do teste de Tukey, para a avaliação intergrupos, feita de maneira independente para cada uma das condições experimentais definidas.

\section{RESULTADOS}

Os 3 grupos (GC: 55,5 $\pm 9,72$ anos; GD: 54,4 $\pm 7,76$ anos; GNP: 60,4 $\pm 5,35$ anos) foram semelhantes quanto a idade, não apresentando diferenças significativas $(p=0,19)$. O IMC do grupo controle foi o menor ( $\mathrm{p}=0,0021)\left(\mathrm{GC}: 26,19 \pm 1,55 \mathrm{~kg} / \mathrm{m}^{2}\right.$; GD 28,18 $\pm 1,33 \mathrm{~kg} / \mathrm{m}^{2}$; GNP: $27,96 \pm 2,20 \mathrm{~kg} / \mathrm{m}^{2}$ ) em decorrência da maior massa corporal dos demais grupos, uma vez que não foram encontradas diferenças significativas na estatura dos grupos $(\mathrm{p}=0,64)$.

O tempo médio de diabetes foi 4,1 anos para o grupo GD e 10,7 para o GNP. A média da pontuação do MNSI foi de 9,8 pontos no grupo GNP.

As Figuras 1 e 2 apresentam os gráficos das oscilações no plano frontal e sagital, nas condições com e sem visão. Nos dois planos, o grupo GNP apresentou as maiores oscilações nas duas condições em relação aos demais grupos, com diferenças significativas de $\mathrm{p}=0,0001$. No entanto, não foram verificadas significâncias entre GC e GD, em nenhuma condição nos dois planos $(p>0,05)$. 
$\mathrm{Na}$ Figura 3 estão representados os valores, em módulo, da diferença das oscilações, com e sem visão, obtidas em um mesmo plano. Foi evidenciada uma maior diferença entre as condições no GNP em relação aos outros grupos, tanto para oscilação no plano frontal, como no sagital ( $p=0,0001)$.

\section{DISCUSSÃO}

A metodologia desenvolvida no presente trabalho visou avaliar o equilíbrio estático de indivíduos diabéticos neuropatas, com e sem informação visual, e compará-lo com o equilíbrio de indivíduos diabéticos não neuropatas e indivíduos sem DM. O método utilizado mostrou-se de fácil aplicação na avaliação do equilíbrio estático, como descreveram Cardoso ${ }^{30}$, Guimarães ${ }^{31}$ e Baraúna et al. ${ }^{32}$, deixando claro que se trata de um instrumento capaz de avaliar o equilíbrio quantitativamente, ou seja, com maior fidedignidade.

\section{Oscilação plano frontal com visão}

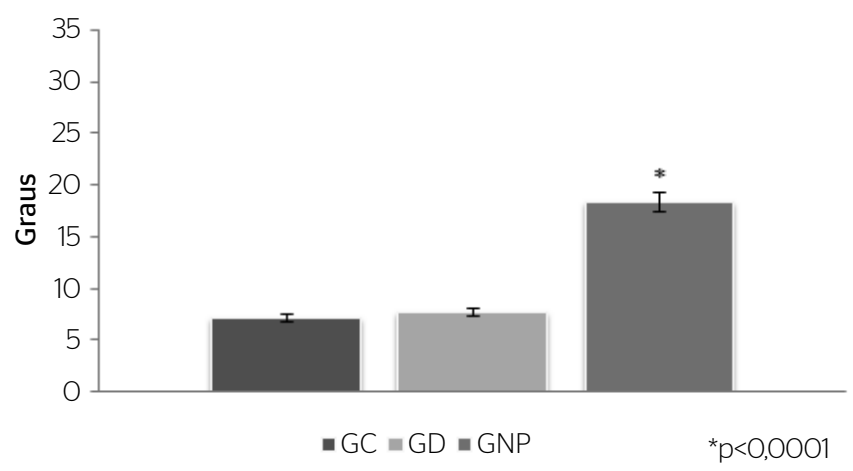

A eleição do teste de Romberg adaptado para avaliação do equilíbrio estático em 30 s é justificada pelos resultados obtidos por Presumido ${ }^{33}$ e Baraúna ${ }^{27}$, os quais demonstraram que as maiores oscilações ocorrem nesse intervalo de tempo.

Analisando os resultados, observamos as maiores oscilações no plano frontal e sagital com e sem visão no grupo GNP (Figuras 1 e 2).

As diferenças estatisticamente significativas encontradas entre o grupo GNP e o grupo GD nos leva a julgar que a neuropatia é o fator responsável pelo déficit de equilíbrio, fato corroborado pela ausência de diferenças entre os grupos GD e GC. Como os grupos estudados possuem médias semelhantes de idade, descarta-se a possibilidade dessas diferenças serem atribuídas ao processo natural de envelhecimento.

Os dados encontrados em nosso estudo também concordam com os de Meyer et al. ${ }^{34}$, que focalizaram o papel da sensação cutânea plantar no controle do equilíbrio estático. Usando uma anestesia para reduzir a sensibilidade da planta do pé, concluíram que a sensação

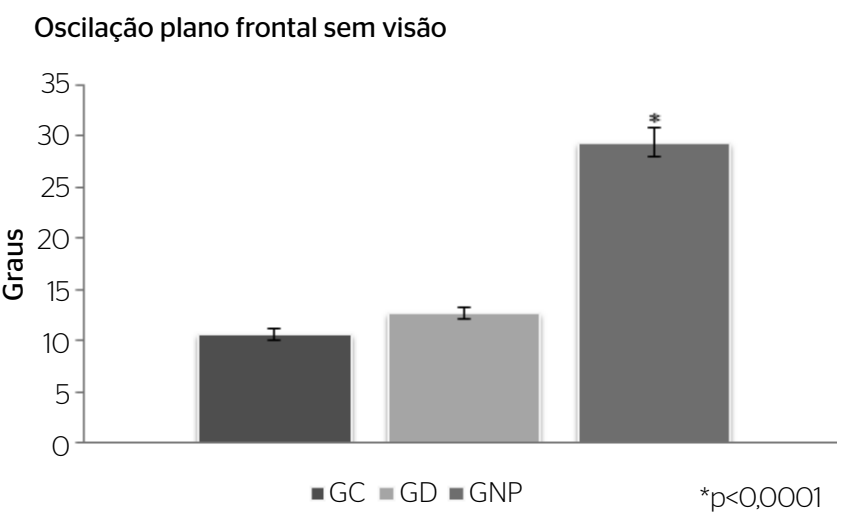

GC: grupo controle; GD: grupo diabetes; GNP: grupo neuropatia diabética

Figura 1. Gráficos das oscilações no plano frontal nas situações com e sem visão

\section{Oscilação plano sagital com visão}

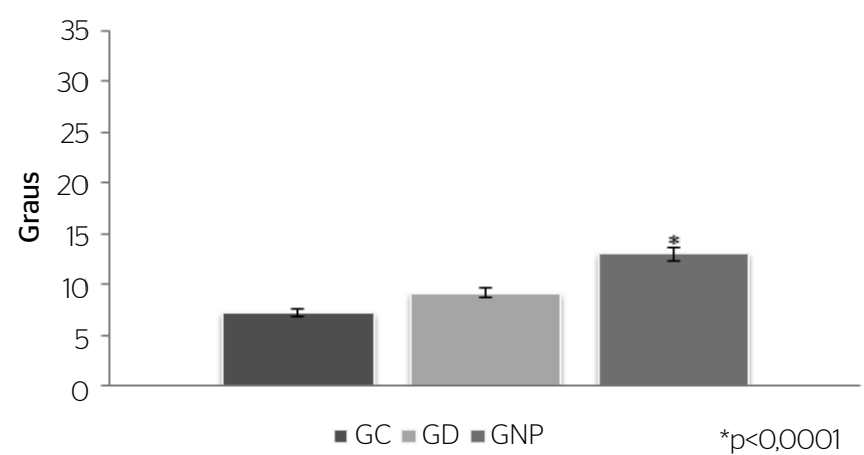

Oscilação plano sagital sem visão

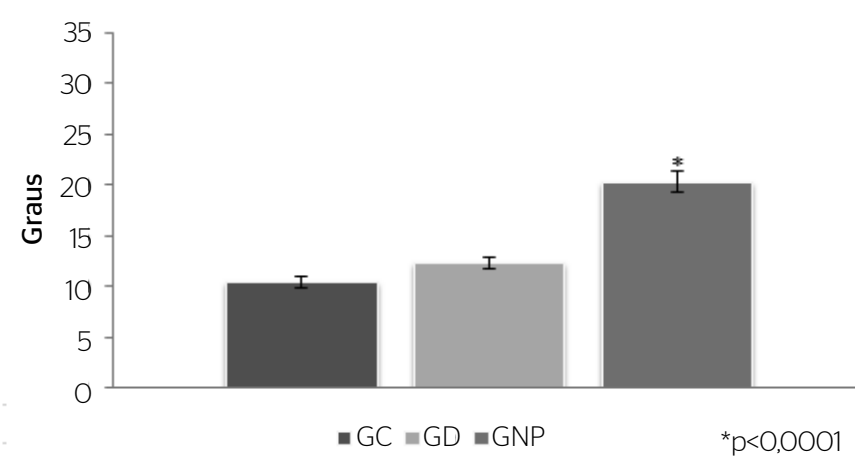




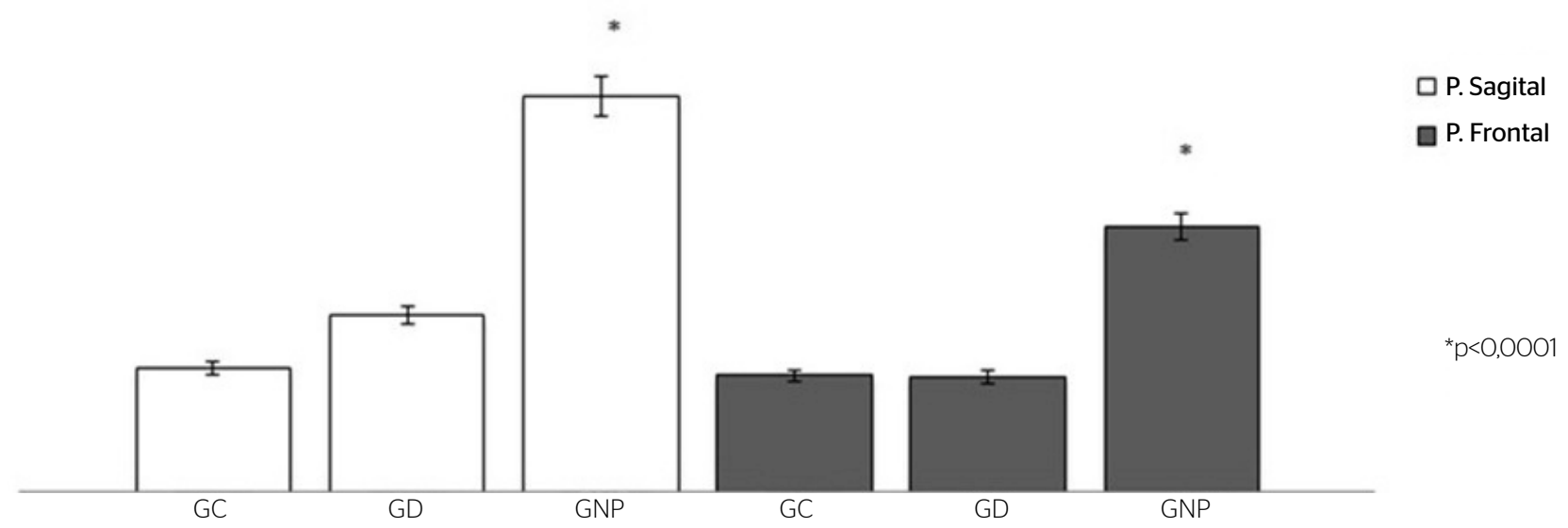

GC: grupo controle; GD: grupo diabetes; GNP: grupo neuropatia diabética; P. Sagital: plano sagital; P. Frontal: plano frontal

Figura 3. Valores em módulo da diferença das oscilações com e sem visão, obtidas no plano sagital e frontal

plantar é importante para a preservação do equilíbrio ortostático normal quando a visão não é permitida.

A remoção da informação visual ocasionou maiores perturbações no equilíbrio do grupo GNP (Figura 3). Tais resultados podem ser compreendidos no estudo de Ahmmed e Mackenzie ${ }^{35}$ que comparou três grupos (diabéticos, diabéticos neuropatas e controle), revelando maior oscilação com os olhos fechados nos diabéticos com neuropatia, demonstrando que o estímulo visual auxilia no equilíbrio postural.

Lord et al. ${ }^{36}$ argumentaram que a sensibilidade periférica é o mais importante sistema sensorial para a manutenção do equilíbrio. Isso explica maiores amplitudes de oscilação encontradas no grupo GNP pelo presente estudo. Fato semelhante foi encontrado por Menz et al. ${ }^{37}$ ao observarem que a alteração de equilíbrio estaria mais relacionada com a alteração da sensibilidade tátil do que a visão.

Emam et al..$^{38}$ avaliaram a estabilidade postural em 54 pacientes diabéticos tipo 2 com neuropatia, utilizando a posturografia dinâmica computadorizada e observaram que houve diferenças significativas no equilíbrio estático entre pacientes neuropáticos em comparação com pacientes não neuropáticos. No entanto, em condições dinâmicas, não houve diferença significativa entre os dois grupos. Segundo o autor, esses resultados podem refletir o maior comprometimento do sistema somatossensorial em relação aos sistemas vestibulares e/ou visuais no equilíbrio em pacientes diabéticos.

Diferente dos resultados obtidos no presente trabalho, Nozabielii ${ }^{39}$ não evidenciou diferenças significativas ao comparar o equilíbrio estático de indivíduos neuropatas com um grupo controle. Porém, as metodologias empregadas foram diferentes. A autora atribuiu seus resultados ao maior peso corpóreo dos neuropatas e a não supressão do estímulo visual, o que possivelmente mascarou a instabilidade No presente estudo, entretanto, o grupo GNP demonstrou maiores oscilações, mesmo apresentando as maiores massas corporais e utilizando a informação visual.

As limitações deste estudo incluem a falta de controle de fatores, como nível de atividade física e alterações visuais, tendo em vista a influência desses na avaliação do equilíbrio corporal.

\section{CONCLUSÃO}

Apesar da amostra pequena, constatou-se que a NP influencia negativamente na manutenção do equilíbrio estático e o diabetes, por si, não teve efeitos sobre a instabilidade postural. A visão auxilia no controle postural e sua remoção aumentou ainda mais o desequilibrio, principalmente nos neuropatas, evidenciando que o déficit sensitivo é a principal causa das instabilidades posturais nesse grupo.

\section{REFERÊNCIAS}

1. Wild S, Roglic G, Green A, Sicree R, King H. Global prevalence of diabetes: estimates for the year 2000 and projections for 2030. Diabetes Care. 2004:27(5):1047-53. 
2. Sacco ICN, Sartor CD, Gomes AA, João SMA, Cronfli R. Avaliação das perdas sensório-motoras do pé e tornozelo decorrentes da neuropatia diabética. Rev Bras Fisioter. 2007;11(1):27-33.

3. Leonard DR, Farooqi MH, Myers S. Restoration of sensation, reduced pain, and improved balance in subjects with diabetic peripheral neuropathy: a double blind, randomized, placebo controlled study with monochromatic near infrared treatment. Diabetes Care. 2004;27(1):168-72.

4. Said G. Focal and multifocal diabetic neuropathies. Arq Neuropsiquiatr. 2007;65(4):1272-8

5. Tapp R, Shaw J, Courten M, Dunstan D, Welborn T, Zimmet P. Foot complications in type 2 diabetes: an Australian population based study. Diabet Med. 2003;20(2):105-13.

6. Bacarin TA, Sacco IC, Hennig EM. Plantar pressure distribution patterns during gait in diabetic neuropathy patients with a history of foot ulcers. Clinics (São Paulo). 2009;64(2):113-20.

7. Anjos DMC. Verificação da relação entre alterações de pressão plantar e o equilíbrio em pacientes diabéticos por meio de parâmetros baropodométricos e estabilométricos [dissertação]. São José dos Campos: Universidade do Vale do Paraíba; 2006.

8. Kandel ER, Schwartz JH, Jessell TM. Principles of neural science. 3a ed. Norwalk: Appleton \& Lange; 1991.

9. Tribastone F. Tratado de exercícios corretivos aplicados à reeducação motora postural. São Paulo: Manole; 2001.

10. Baraúna MA, Duarte F, Sanchez HM, Canto RST, Malusá S, CampeloSilva CD, et al. Avaliação do equilíbrio estático em indivíduos amputados de membros inferiores através da biofotogrametria computadorizada. Rev Bras Fisioter. 2006;10(1):83-90.

11. Lima FD. Avaliação da influência do salto no equilíbrio estático em diabéticos tipo 2 através da baropodometria computadorizada [dissertação]. São José dos Campos: Universidade do Vale do Paraíba; 2007.

12. Richardon JK. The clinical identification of peripheral neuropathy among older persons. Arc Phys Med Rehabil. 2002;83(11):1553-8.

13. Dingwell JB, Cusumano JP, Sternad D, Cavanaghet PR. Slower speeds in patients with diabetic neuropathy lead to improved local dynamic stability of continuous overground walking. J Biomech. 2000;33(10):1269-77.

14. Boucher P, Teasdale N, Courtemanche R, Bard C, Fleury M. Postural stability in diabetic polyneuropathy. Diabetes Care. 1995:18(5):638-45.

15. Lafond D, Corriveau H, Prince F. Postural control mechanisms during quiet standing in patients with diabetic sensory neuropathy. Diabetes Care. 2004;27(1):173-8.

16. Uccioli L, Giacomini PG, Monticone G, Magrin A, Durola L, Bruno E, et al. Body sway in diabetic neuropathy. Diabetes Care. 1995;18(3):339-44.

17. Bonnet C, Carello C, Turvey MT. Diabetes and postural stability: review and hypotheses. J Mot Behav. 2009;41(2):172-90.

18. Fulk GD, Robinson CJ, Mondal S, Storey CM, Hollister AM. The effects of diabetes and/or peripheral neuropathy in detecting short postural perturbations in mature adults. J Neuroeng Rehabil. 2010;7:44.

19. Allet L, Armand Sp, de Bie RA, Pataky Z, Aminian K, Herrmann FR, et al. Gait alterations of diabetic patients while walking on different surfaces. Gait Posture. 2009;29(3):488-93.

20. Oppenheim U, Kohen-Raz R, Alex D, Kohen-Raz A, Azarya M. Postural characteristics of diabetic neuropathy. Diabetes Care. 1999:22(2):328-32.
21. Centomo H, Termoz N, Savoie S, Beliveau L, Prince F. Postural control following a self-initiated reaching task in type 2 diabetic patients and age-matched controls. Gait Posture. 2007:25(4):509-14.

22. American Diabetes Association. Diagnosis and classification of diabetes mellitus. Diabetes Care. 2009;32(1):62-7.

23. Grupo de trabalho sobre pé diabético. Consenso internacional sobre pé diabético. Brasília: Secretaria de Estado de Saúde; 2011.

24. Ulhoa LS, Lima RCO, Cunha VNC, Gomes EB, Campbell CSG, Pedrosa HC. Mobilidade articular de idosos diabéticos e não diabéticos e influência da fisioterapia. Fisioter Mov. 2011;24(1):99-106.

25. University of Michigan Health System, Michigan Diabetes Research and Training Center (2009) MNSI - Michigan Neuropathy Screening Instrument [cited 2011 Mar 10]. Available from: http://www.med.umich. edu/mdrtc/profs/survey.html

26. Moghtaderi A, Bakhshipour A, Rashidi H. Validation of Michigan neuropathy screening instrument for diabetic peripheral neuropathy. Clin Neurol Neurosurg. 2006;108(5):477-81.

27. Baraúna MA. Estudo comparativo entre a avaliação do equilíbrio estático de indivíduos amputados e não amputados [Tese]. Lisboa (Portugal): Universidade Técnica de Lisboa; 1997.

28. Meneghetti CHZ, Blascovi-Assis SM, Deloroso FT, Rodrigues GM. Avaliação do equilíbrio estático de crianças e adolescentes com síndrome de Down. Rev Bras Fisioter. 2009;13(5):230-5.

29. Portal do software para avaliação postural [homepage na internet]. São Paulo: Incubadora Virtual FAPESP [cited 2011 Mar 3]. Available from: http://sapo.incubadora.fapesp.br/portal/projeto/FrontPage

30. Cardoso FAG. Avaliação do equilíbrio estático em pacientes diabéticos portadores de neuropatia autonômica, através da fotogrametria computadorizada [Dissertação]. Uberlândia: UNITRI Centro Universitário do Triângulo; 1999.

31. Guimarães EA. Avaliação do equilíbrio estático de indivíduos normais através da biofotogrametria computadorizada e da oscilometria [Dissertação]. Uberlândia: UNITRI - Centro Universitário do Triângulo; 2003.

32. Baraúna MA, Barbosa SRM, Canto RST, Silva RAV, Silva CDC, Baraúna KMP. Estudo do equilíbrio estático de idosos e sua correlação com quedas. Fisioter Bras. 2004:5(2):136-41.

33. Presumido LMB, Baraúna MA, Ferreira C, Silva KC. Estudo comparativo entre o equilíbrio estático de indivíduos sedentários e não sedentários do sexo feminino. Ícone. 1995;3(2):39-62.

34. Meyer PF, Oddsson LIE, De Luca CJ. The role of plantar cutaneous sensation in unperturbed stance. Exp Brain Res. 2004:156(4):505-12.

35. Ahmmed AU, Mackenzie IJ. Posture changes in diabetes mellitus. J Laryngol Otol. 2003;117(5):358-64.

36. Lord SR, Clark RD, Webster LW. Postural stability and associated physiological factors in a population of aged persons. J Gerontol. 1991;46(3):69-76.

37. Menz HB, Lord SR, George RST, Fitzpatrick RC. Walking stability and sensorimotor function in older people with diabetic peripheral neuropathy. Arch Phys Med Rehabil. 2004:85(2):245-52.

38. Emam AA, Gad AM, Ahmed MM, Assal HS, Mousa SG. Quantitative assessment of posture stability using computerised dynamic posturography in type 2 diabetic patients with neuropathy and its relation to glycaemic control. Singapore Med J. 2009;50(6):614-8.

39. Nozabieli AJL. Avaliação da distribuição da pressão plantar e equilíbrio de diabéticos neuropatas [Dissertação]. Presidente Prudente: Universidade Estadual Paulista; 2010 